\title{
NESTED SIMILARITY IN MATRIX THEORY
}

AnNRAOI DE PAOR, MRIA

School of Electrical and Electronic Engineering, University College Dublin, Belfield, Dublin 4

and

TOMÁs E WARD*

Insight SFI Research Centre for Data Analytics, Dublin City University, Glasnevin, Dublin 9

[Accepted 17 December 2019.]

\section{ABSTRACT}

We explore the problem of making two matrices similar while at the same time submatrices are similar. An approach is adopted based on ideas from state variable feedback and control theory resulting in an easily implementable solution to the problem. The method is perfectly general and puts no constraints on the matrices other than they are real.

\section{Derivation}

We consider the problem of nested similar matrices. We begin with a given $A$ and $B$, real $n \times n$, we want $B$ similar to $A$, i.e.

$$
B=N A N^{-1}
$$

simultaneously if we partition $A$ and $B$ as follows:

$$
A=\left[\begin{array}{l|l}
a_{11} & a_{1}^{T} \\
\hline a_{2} & A_{22}
\end{array}\right], B=\left[\begin{array}{l|l}
b_{11} & b_{1}^{T} \\
\hline b_{2} & B_{22}
\end{array}\right]
$$

with $a_{11} 1 \times 1, a_{1}^{T} 1 \times(n-1), a_{2}(n-1) \times 1, A_{22}(n-1) \times(n-1)$, and similarly for $B$ we want $B_{22}$ similar to $A_{22}$, i.e.,

$$
B_{22}=M A_{22} M^{-1}
$$

We want to explore the conditions under which this is possible, i.e. that matrices

\footnotetext{
*Corresponding author, e-mail: tomas.ward@dcu.ie

${ }^{1}$ This problem was proposed to the first author by the late Professor J. O. Scanlan, long term editor of the International Journal of Circuit Theory and former President of the Royal Irish Academy.
} 
and submatrices can be similar. To begin, we write (1.1) as $N A=B N$, i.e.

$$
\left[\begin{array}{c|c}
n_{11} \mid n_{1}^{T} \\
\hline n_{2} \mid N_{22}
\end{array}\right]\left[\frac{a_{11} \mid a_{1}^{T}}{a_{2} \mid A_{22}}\right]=\left[\frac{b_{11} \mid b_{1}^{T}}{b_{2} \mid M A_{22} M^{-1}}\right]\left[\frac{n_{11} \mid n_{1}^{T}}{n_{2} \mid N_{22}}\right]
$$

We take it that $A$ and $M$ are given and that we have to find $b_{11}, b_{1}^{T}, b_{2}$ (and thus $B_{22}$ )and $n_{11}, n_{1}^{T}, n_{2}, N_{22}$. We begin by writing the equation for the $(2,1)$ blocks in (1.3):

$$
\begin{gathered}
n_{2} a_{11}+N_{22} a_{2}=b_{2} n_{11}+M A_{22} M^{-1} n_{2} \\
\therefore b_{2} n_{11}=\left[a_{11} I-M A_{22} M^{-1}\right] n_{2}+N_{22} a_{2} \\
\therefore b_{2}=\frac{1}{n_{11}}\left\{\left[a_{11} I-M A_{22} M^{-1}\right] n_{2}+N_{22} a_{2}\right\}
\end{gathered}
$$

This defines $b_{2}$ in terms of $n_{11}, n_{2}$ and $N_{22}$. Note that we choose

$$
n_{11} \neq 0
$$

We now look at the $(2,2)$ block in $(1.3)$

$$
n_{2} a_{1}^{T}+N_{22} A_{22}=b_{2} n_{1}^{T}+M A_{22} M^{-1} N_{22}
$$

Using (1.6) to substitute for $b_{2}$, this gives

$$
\begin{array}{r}
M A_{22} M^{-1} N_{22}-N_{22}\left[A_{22}-\frac{a_{2} n_{1}^{T}}{n_{11}}\right]=n_{2} a_{1}^{T}-\frac{1}{n_{11}}\left[a_{11} I-M A_{22} M^{-1}\right] n_{2} n_{1}^{T} \\
\Rightarrow M A_{22} M^{-1} N_{22} I-I N_{22}\left[A_{22}-\frac{a_{2} n_{1}^{T}}{n_{11}}\right]=n_{2} a_{1}^{T}-\frac{1}{n_{11}}\left[a_{11} I-M A_{22} M^{-1}\right] n_{2} n_{1}^{T}
\end{array}
$$

To address the solution of this we write the $N_{22}$ in terms of its columns.

$$
N_{22}=\left[\left(N_{22}\right)_{1},\left(N_{22}\right)_{2}, \ldots,\left(N_{22}\right)_{n-1}\right]
$$

and rearrange this as a single column of dimension $(n-1)^{2} \times 1$ :

$$
n=\left[\begin{array}{c}
\left(N_{22}\right)_{1} \\
\left(N_{22}\right)_{2} \\
\vdots \\
\left(N_{22}\right)_{n-1}
\end{array}\right]
$$

Similarly we introduce the notation

$$
F=n_{2} a_{1}^{T}-\frac{1}{n_{11}}\left[a_{11} I-M A_{22} M^{-1}\right] n_{2} n_{1}^{T}
$$


and rewrite $F$ in terms of its columns as

$$
F=\left[(F)_{1},(F)_{2}, \ldots, F_{n-1}\right]
$$

defining the $(n-1)^{2} \times 1$ column $f$ as

$$
f=\left[\begin{array}{c}
(F)_{1} \\
(F)_{2} \\
\vdots \\
(F)_{n-1}
\end{array}\right]
$$

We now convert (1.10) to Kronecker product notation:

$$
\left[I \otimes M A_{22} M^{-1}-\left[A_{22}-\frac{a_{2} n_{1}^{T}}{n_{11}}\right]^{T} \otimes I\right] n=f
$$

Now, as will be shown later, the $(n-1)^{2} \times(n-1)^{2}$ matrix on the left hand side of (1.16) is nonsingular if and only if $M A_{22} M^{-1}$ and $\left[A_{22}-\frac{a_{2} n_{1}^{T}}{n_{11}}\right]^{T}$ have no eigenvalues in common, i.e. if and only if $A_{22}$ and $A_{22}-\frac{a_{2} n_{1}^{T}}{n_{1}}$ have no eigenvalue in common. Hence we invoke state variable feedback theory $\lceil 1]$ provided $\left(A_{22}, a_{2}\right)$ form a controllable pair, i.e. provided

$$
\operatorname{det}\left[a_{2}, A_{22} a_{2},\left(A_{22}\right)^{2} a_{2}, \ldots,\left(A_{22}\right)^{n-2} a_{2}\right] \neq 0
$$

It is possible to choose the $1 \times(n-1)$ row $\frac{n_{1}^{T}}{n_{11}}$ so that $\left[A_{22}-\frac{a_{2} n_{1}^{T}}{n_{11}}\right]$ has any desired eigenvalues, subject only to complex pairing (since the matrices are real). We specify any $n_{11} \neq 0$ and select $n_{1}^{T}$ accordingly.

Now lets look again at $F$.

$$
F=n_{2} a_{1}^{T}-\frac{1}{n_{11}}\left[a_{11} I-M A_{22} M^{-1}\right] n_{2} n_{1}^{T}
$$

Having specified $n_{11}$ and completed $n_{1}^{T}$ so that $A_{22}$ and $A_{22}-\frac{a_{2} n_{1}^{T}}{n_{11}}$ have no eigenvalues in common, all we need to do now is choose $n_{2},[(n-1) \times 1]$, so that $F \neq 0$, i.e. $f \neq 0$ in (1.16). We then solve (1.16) for $n$, i.e. for $N_{22}$ from (1.12).

We have now got $n_{11}, n_{1}^{T}, n_{2}$ and $N_{22}$, i.e. we have the $n \times n$ matrix $N$. We must check that $\operatorname{det} N \neq 0$. We can write

$$
\operatorname{det} N=n_{11} \cdot \operatorname{det}\left[N_{22}-\frac{n_{2} n_{1}^{T}}{n_{11}}\right]
$$

, so that the extra condition here is

$$
\operatorname{det}\left[N_{22}-\frac{n_{2} n_{1}^{T}}{n_{11}}\right] \neq 0
$$


Note that we have $b_{2}$ from equation (1.6)

Finally, we solve for $b_{11}$ and $b_{1}^{T}$ from the equation

$$
\left[b_{11}, b_{1}^{T}\right] N=\left[n_{11}, n_{1}^{T}\right] A
$$

The first row of $B N$ is the first row of $N A$. This concludes the solution. It is worth highlighting that in many applications the matrix A may be assumed to have $n$ distinct eigenvalues. In such a case it suffices that $\mathrm{A}$ and $\mathrm{B}=\left[\frac{b_{11} \mid b_{1}^{T}}{b_{2} \mid M A_{22} M^{-1}}\right]$ have the same characteristic polynomial. Note we make use of the observation that $b_{11}=a_{11}$ when a solution exists. The characteristic polynomial condition in this case can then be expressed through the use of Schur complements as follows:

$$
\operatorname{tr}\left(a_{1}^{T} A_{22}^{j} a_{2}\right)=\operatorname{tr}\left(b_{1}^{T} M A_{22}^{j} M^{-1} b_{2}\right)
$$

for $j=0,1, \ldots, n-2$. From such a formulation a simple solution can be seen in the form of $B_{22}=M A_{22} M^{-1}, b_{1}^{T}=a_{1}^{T} M^{-1}, b_{2}=M a_{2}$.

\section{EXAMPLE}

We assume $\mathrm{A}$ and $\mathrm{M}$ are given. In this example

$$
A=\left[\begin{array}{ccc}
1 & 5 & -3 \\
5 & -4 & 15 \\
-7 & 2 & 9
\end{array}\right], M=\left[\begin{array}{cc}
-5 & 6 \\
23 & -5
\end{array}\right]
$$

where $\mathrm{M}$ has been chosen to be invertible. Next we specify any $n_{11} \neq 0$ and select $n_{1}^{T}$ accordingly.

So let us arbitrarily choose $n_{11}=5$

$n_{1}^{T}$ is now arbitrarily chosen subject to the constraint that $A_{22}$ and $A_{22}-\frac{a_{2} n_{1}^{T}}{n_{11}}$ have no eigenvalues in common, for example:

$$
n_{1}^{T}=\left[\begin{array}{ll}
3 & -7
\end{array}\right]
$$

Examination of the corresponding eigenvalues confirms the appropriateness of this choice of $n_{1}^{T}$. We now choose $n_{2},[(n-1) \times 1]$, so that $F \neq 0$, for example with:

$$
n_{2}=\left[\begin{array}{l}
5 \\
6
\end{array}\right]
$$

This yields

$$
F=\left[\begin{array}{cc}
16.1965 & 5.5416 \\
224.3363 & -471.4513
\end{array}\right]
$$

We use $\mathrm{F}$ then to solve (1.16) for $n$, i.e. for $N_{22}$ from (1.12).

${ }^{2}$ We would like to thank an anonymous reviewer for this insight 
We have now got $n_{11}, n_{1}^{T}, n_{2}$ and $N_{22}$, i.e. we have the $n \times n$ matrix $N$ having checked that $\operatorname{det} N \neq 0$. $\mathrm{N}$ in this case is revealed as

$$
N=\left[\begin{array}{ccc}
5 & 3 & -7 \\
5 & -0.63703 & -1.66247 \\
6 & 11.07589 & -15.44987
\end{array}\right]
$$

We calculate $b_{2}$ from equation (1.6) and the remaining unknown elements of $B$ from $(1.20)$

This reveals $B$ as

$$
B=\left[\begin{array}{ccc}
1 & 12.379 & 0.35083 \\
4.62494 & -2.85841 & 0.76991 \\
-32.07305 & 56.54867 & 7.85841
\end{array}\right]
$$

and concludes the calculation.

In summary this worked example demonstrates how for a given $A$ and $M$ the method described in this paper provides a means for calculating $B$ and $N$ such that:

$$
B=N A N^{-1}
$$

and simultaneously with $A$ and $B$ partitioned as follows:

$$
A=\left[\begin{array}{l|l}
a_{11} & a_{1}^{T} \\
\hline a_{2} & A_{22}
\end{array}\right], B=\left[\begin{array}{l|l}
b_{11} & b_{1}^{T} \\
\hline b_{2} & B_{22}
\end{array}\right]
$$

with $a_{11} 1 \times 1, a_{1}^{T} 1 \times(n-1), a_{2}(n-1) \times 1, A_{22}(n-1) \times(n-1)$, that $B_{22}$ is similar to $A_{22}$, i.e.,

$$
B_{22}=M A_{22} M^{-1}
$$

\section{APPENDIX}

Nonsingularity of the $(n-1)^{2} \times(n-1)^{2}$ matrix, $\left[I \otimes M A_{22} M^{-1}-\left[A_{22}-\frac{a_{2} n_{1}^{T}}{n_{11}}\right]^{T} \otimes I\right]$. To simplify the notation let's write this as $[I \otimes P-Q \otimes I]$.

Now let $V_{i}$ be an eigenvector of $P$ belonging to the eigenvalue $\lambda_{i}$ and $W_{j}$ be an eigenvector of $Q$ belonging to the eigenvalue $\rho_{i}$. Now consider

$$
\begin{array}{r}
{[I \otimes P-Q \otimes I]\left[W_{j} \otimes V_{i}\right]=[I \otimes P]\left[W_{j} \otimes V_{i}\right]-[Q \otimes I]\left[W_{j} \otimes V_{i}\right]} \\
=\left[I W_{j} \otimes P V_{i}\right]-\left[Q W_{j} \otimes I V_{i}\right] \\
=\left[W_{j} \otimes \lambda_{i} V_{i}\right]-\left[\rho_{i} W_{j} \otimes V_{i}\right] \\
=\left(\lambda_{i}-\rho_{i}\right)\left[W_{j} \otimes V_{i}\right]
\end{array}
$$

Therefore $W_{j} \otimes V_{i}$ is an eigenvector of $[I \otimes P-Q \otimes I]$ belonging to the eigenvalue $\lambda_{i}-\rho_{i}$. A matrix is singular if and only if it has 0 as an eigenvalue. Therefore 
$[I \otimes P-Q \otimes I]$ is nonsingular if and only if $P$ and $Q$ have no eigenvalues in common, i.e. if and only if $M A_{22} M^{-1}$ and $\left[A_{2}-\frac{a_{2} n_{1}^{T}}{n_{11}}\right]^{T}$ have no eigenvalues in common, i.e. if and only if $A_{22}$ and $A_{2}-\frac{a_{2} n_{1}^{T}}{n_{11}}$ have no eigenvalues in common.

As mentioned, provided $\left(A_{22}, a_{2}\right)$ is a controllable pair, $\frac{n_{1}^{T}}{n_{11}}$ can always be chosen to ensure this. Interestingly and relevant, a recent result [2] examines conditions under which the Sylvester equation $P X-X Q=Z$ where $P$ and $Q$ may have some common eigenvalues, is solvable.

\section{REFERENCES}

[1] H. M. Power, "A new result on eigenvalue assignment by means of dyadic output feedback," International Journal of Control, vol. 21, pp. 149-158, Jan 1975.

[2] N. C. Dinčić, "Solving the sylvester equation $\mathrm{ax}-\mathrm{xb}=\mathrm{c}$ when $\sigma(a) \cap \sigma(b) \neq \emptyset$," Electronic Journal of Linear Algebra, vol. 35, pp. 1-23, 2019.

${ }^{3}$ Again we acknowledge the valuable contribution of an anonymous reviewer for drawing our attention to this result 